\title{
Eosinophilic Granulomatosis with Polyangiitis and Atopy: A Case Report
}

\author{
Alejandro Raúl Gratacós Gómez¹, Oscar Marcelo González Jimenez², \\ Juana Bautista Joyanes Romo ${ }^{2}$, Alberto Palacios Cañas ${ }^{2}$, Rosa Garcia Rodriguez ${ }^{2}$ \\ and Elisa Gomez Torrijos ${ }^{2 *}$ (1) \\ ${ }^{1}$ Universidad de Castilla La Mancha, Facultad de Medicina, Ciudad Real, Spain; ${ }^{2}$ Allergology Service, Hospital \\ General Universitario de Ciudad Real, Spain
}

\begin{abstract}
A 36-year-old male, diagnosed with atopic dermatitis and allergy to egg white when he was a baby, was diagnosed in adolescence with Bird-Egg Syndrome and seasonal rhinoconjunctivitis with bronchial asthma. Five years later, he developed rhinosinusitis, nasosinusal polyposis and persistent bronchial asthma. At the age of 27, this individual presented with eosinophilic esophagogastroduodenitis and peripheral eosinophilia with a total IgE of 1,989 $\mathrm{KU} / \mathrm{mL}$ and repeatedly tested negative for antineutrophil cytoplasmic antibodies. When he was 31-year-old, the patient was admitted to the hospital due to an exacerbation of his asthma, the onset of fever, bilateral migratory lung infiltrates and maculopapular lesions in the ankles (leukocytoclastic vasculitis). He was also diagnosed with motor-sensory polyneuritis. Finally, this patient met the criteria of allergic granulomatosis with polyangiitis and was treated with Omalizumab (monthly) with effective recovery.
\end{abstract}

Introduction

Atopic eczema (AE) is associated with several comorbidities such as an increased IgE-dependent allergic sensitization, increased infection rates, mental disorders and the development of various autoimmune diseases. ${ }^{1}$ Only some children with AE develop bronchial asthma (BA) and not all BA cases are preceded by AE. The impaired skin barrier associated with $\mathrm{AE}$ facilitates sensitization, which increases the risk for systemic allergic responses. ${ }^{2}$

There are multi-systemic autoimmune diseases that likely involve a complex interaction between genetic and environmental factors. These disorders can lead to an inflammatory response that is mediated mostly by T-lymphocytes (TH2), but also on occasion by eosinophils and B-lymphocytes. ${ }^{3}$ In addition to the skin, some epithelial barrier defects may affect other organs or the airways, which can trigger rhinitis and/or BA. Such defects can also affect epithelial surfaces of the gastrointestinal organs, triggering IgE-mediated food al-

Keywords: Atopic dermatitis; Bronchial asthma; Food allergy; Vasculitis; Polyneuritis; Eosinophilia granulomatosis.

Abbreviations: AE, atopic eczema; BA, bronchial asthma; EGPA, eosinophilic granulomatosis with polyangiitis; ANCA, anti-neutrophil cytoplasmic antibody.

Received: December 18, 2020; Revised: January 12, 2021; Accepted: January 20, 2021 *Correspondence to: Elisa Gómez Torrijos, Allergology Service, Hospital General Universitario de Ciudad Real, C/Obispo Rafael Torija S/N, 13005 Ciudad Real, Spain. ORCID: http://orcid.org/0000-0002-4532-2498. Tel: +34-626-356825, E-mail: egomezt.cr@gmail.com

How to cite this article: Gratacós Gómez AR, González Jimenez OM, Joyanes Romo JB, Palacios Cañas A, Garcia Rodriguez R, Gomez Torrijos E. Eosinophilic Granulomatosis with Polyangiitis and Atopy: A Case Report. Exploratory Research and Hypothesis in Medicine 2020;000(000):000-000. doi: 10.14218/ERHM.2020.00078. lergies, and much less frequently, IgE-non mediated food allergies. ${ }^{2}$ $\mathrm{AE}$ is also epidemiologically associated (23\% to $91 \%)$ with several autoimmune diseases that cause autoreactive $\operatorname{IgE}$ secretion. ${ }^{1}$ Eosinophilic granulomatosis with polyangiitis (EGPA) is a multi-systemic disorder belonging to the larger classification of small vessel anti-neutrophil cytoplasmic antibody (ANCA)-associated vasculitis. ${ }^{3}$ This case report describes a patient who has suffered from multiple atopic diseases since childhood which are associated in adulthood with various symptoms due to multi-organ involvement.

\section{Case report}

We report the case of a 36-year-old male who was diagnosed during the first months of his life with $\mathrm{AE}$ and subsequently with an allergy to egg-white proteins. When he was a teenager, the patient was diagnosed with Egg-Bird Syndrome due to an allergy to proteins (alpha-livetins) contained in egg yolk, bird feather and poultry meat. The patient was also diagnosed with seasonal rhinoconjunctivitis with BA due to an allergy to grass and olive pollens.

Despite being treated with pollen immunotherapy, the patient's seasonal respiratory allergy worsened and, after 7-8 years, evolved into rhinosinusitis, nasosinusal polyposis and persistent BA throughout the year. At the age of 27, the patient started experiencing dysphagia, abdominal pain and diarrhea. After performing an esophagogastroduodenoscopy with biopsies, he was diagnosed with eosinophilic esophagogastroduodenitis since he had more than 15 eosinophils/high power field in the esophagus, and more than 40 eosinophils/high power field in the stomach and duodenum. Periph- 


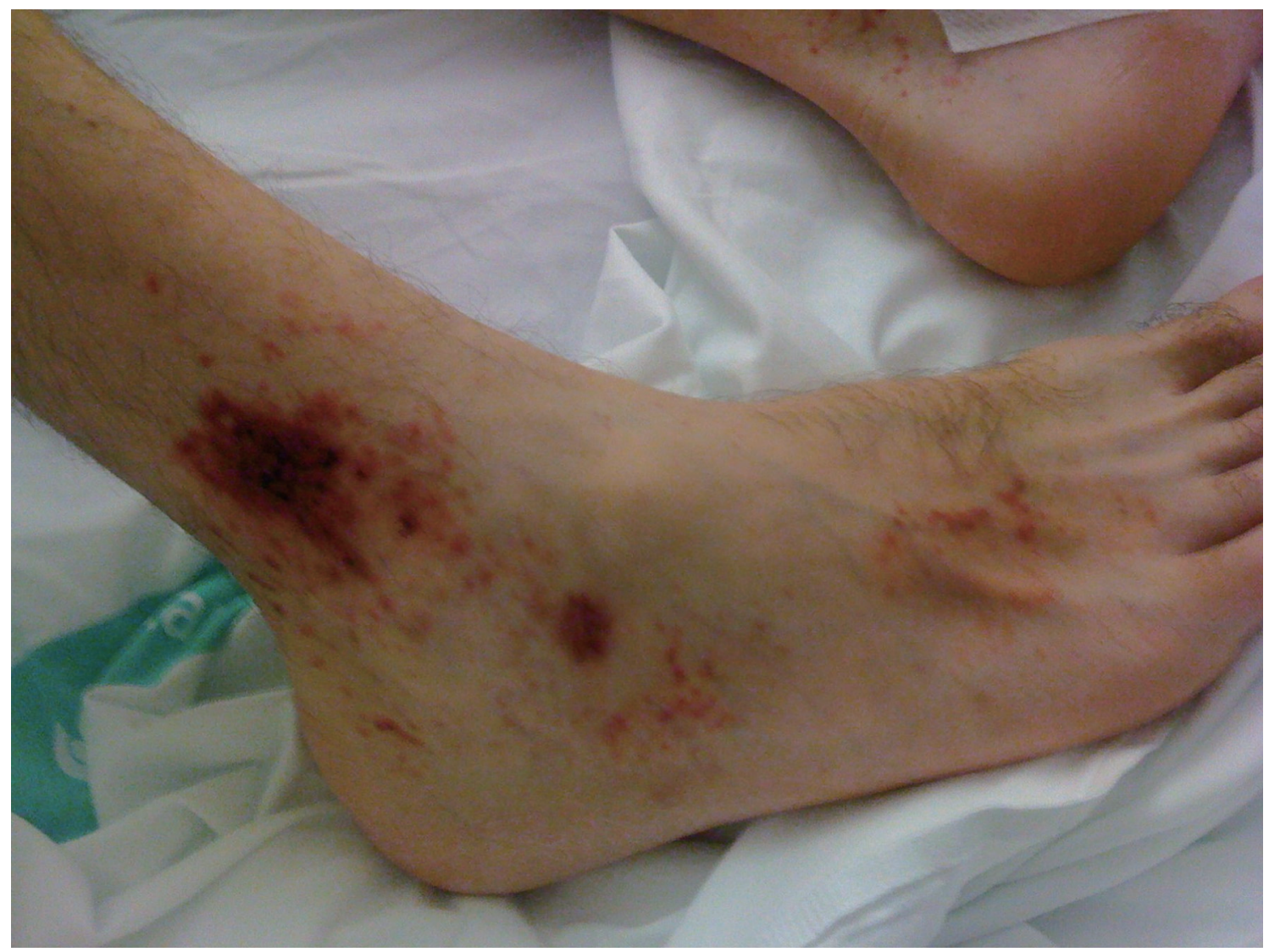

Fig. 1. Leukocytoclastic vasculitis in the external area of the distal third of the right leg and foot.

eral eosinophilia (18-35\%) was also detected as his total IgE was $1,989 \mathrm{KU} / \mathrm{mL}$ and his ANCAs were repeatedly negative.

When the patient was 29 years old, his BA was cortico-dependent and he needed $15 \mathrm{mg}$ of deflazacort and $10 \mathrm{mg}$ of montelukast daily, as well as 50/500 ug of salmeterol/fluticasone every 8 hours, to maintain control. 5-years ago, this patient was admitted to a hospital for an exacerbation of his asthma with fever and bilateral migratory lung infiltrates, and was subsequently diagnosed with the aid of lung computed tomography. The patient also exhibited red-vinous maculopapular lesions in his ankles with a histological report indicating leukocytoclastic vasculitis without granulomas (Fig. 1). The blood tests showed intense peripheral blood eosinophilia. Immunoglobulin (with subclasses), liver, cardiac and renal function tests were all within normal ranges. The rheumatoid factor and antinuclear antibodies were also negative.

By reviewing the patient's medical history, we detected that he was diagnosed at the Neurology Department with sensory-motor polyneuritis after being subjected to an electromyography examination. Based on the sequence of his symptoms (Fig. 2), the patient was diagnosed with EGPA. The patient was treated with prednisone $(1 \mathrm{mg} / \mathrm{kg}$ body weight daily for $1 \mathrm{month}), 225 \mathrm{mgs} / \mathrm{month}$ omalizumab (anti-interleukin 5 [IL5] drugs were not marketed in Spain), and $4 \mathrm{mg}$ deflazacort every $48 \mathrm{hrs}$. We attempted to withdraw deflazacort but the BA and sinus exacerbations reappeared. The evolution of the last 3-4 years has been very favorable and the patient only reports $1-2$ exacerbations of asthma each year that are controlled with a treatment regimen of $15 \mathrm{mg}$ of deflazacort daily for 2 weeks. We proposed to the patient to withdraw deflazacort and/or exchange omalizumab for mepolizumab, but the patient did not accept for fear of worsening his condition. For the last several years, the patient has had no digestive symptoms.

\section{Discussion}

Hypersensitivity reactions of the immune system have been broadly categorized into atopic and autoimmune categories. This classification largely depends on whether the antigen triggering the reaction is endogenous (within the body) or exogenous (outside the body), the types of cellular and humoral components involved, and the clinical symptoms. Clinical studies have served to challenge the idea that the atopy and autoimmunity are mutually exclusive. ${ }^{4}$

The epithelium is not only a protective structure but also an important source of cytokines such as thymic stromal lymphopoietin, IL33, and IL25 that may initiate and drive type-2 inflammation. It has also been shown in EGPA in vitro that CD4+ T-cells can produce high concentrations of IL4, IL5 and IL13, molecules that characterize the TH2 immune response. ${ }^{3}$

According to the 1994 Chapel Hill Consensus Conference, EGPA is defined as a granulomatous and eosinophil-rich inflammation that often affects the respiratory tract and a necrotizing vasculitis that predominantly affects small and medium-sized vessels associated with asthma and eosinophilia. ${ }^{3}$ EGPA is formerly known as Churg-Strauss syndrome and was first described in 1951 by Churg and Strauss as a rare disease with clinical and pathological characteristics between those of primary systemic vasculitis and hypereosinophilic disorders. ${ }^{5}$ The 2012 Chapel Hill Consen- 

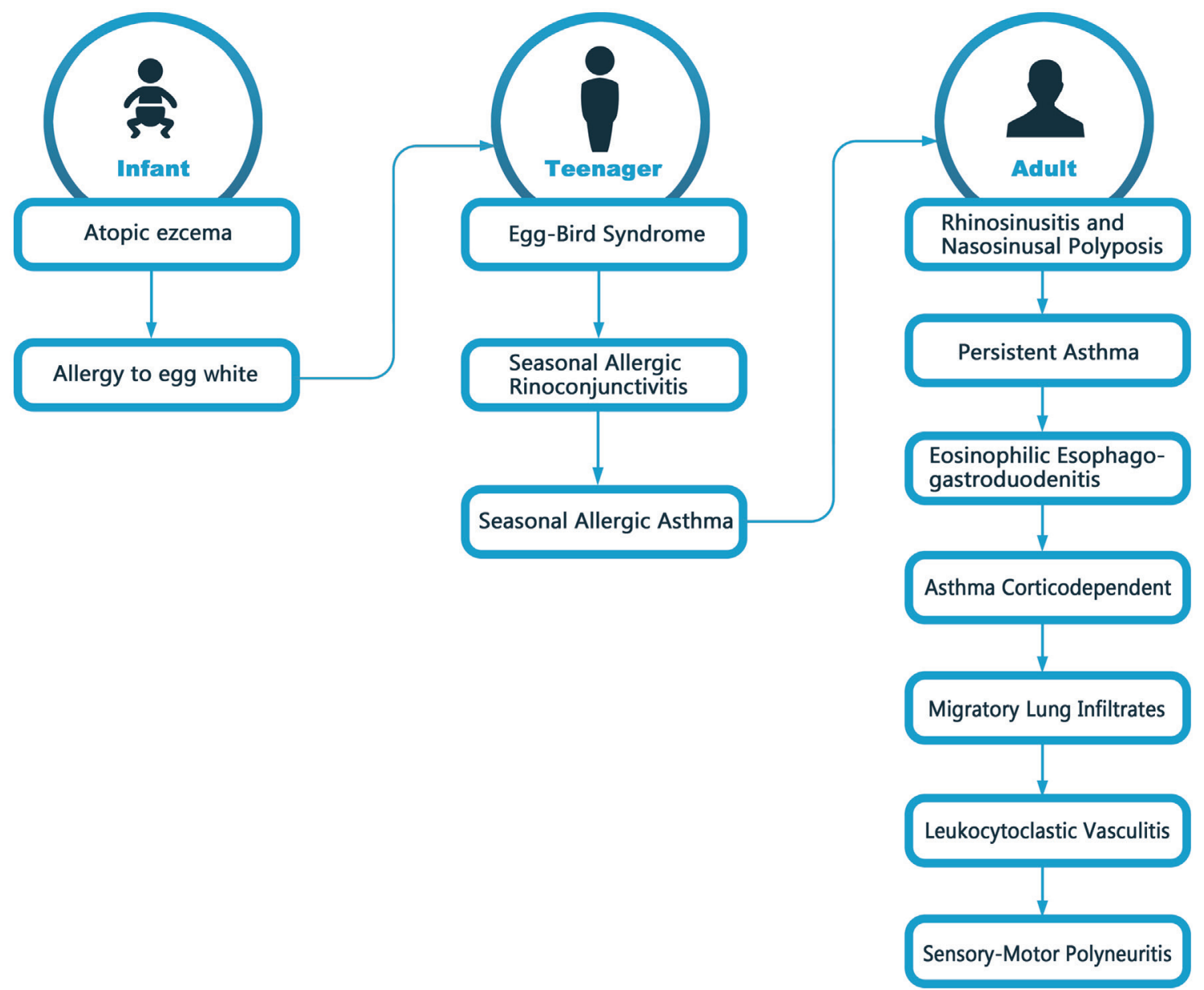

Fig. 2. Chronology in the appearance of symptoms in the patient with Eosinophilic Granulomatosis with Polyangiitis.

sus Conference first reported that ANCA may be found in EGPA, especially in patients with glomerulonephritis. ${ }^{3}$

There are genetically distinct subgroups of EGPA that correspond to the positive and negative ANCA subgroups. ${ }^{6}$ ANCA (especially p-ANCA anti-myeloperoxidase) are present in $30-40 \%{ }^{5}$ or $40-60 \%$ of the patients according to different reports. ${ }^{3}$ Even though these antibodies have always been negative in the patient included in this report, he was ultimately diagnosed with this disease as he met the criteria of the American College Rheumatology $1990 .^{5}$ The rarity of this disease and its unique characteristics, such as eosinophilic inflammation ${ }^{6}$ associated with atopy, delayed the diagnosis of EGPA in our patient. This is likely due to the multisystem affectation of EGPA and the multidisciplinary treatment required. The comorbidities that develop in EGPA patients over time are included in the classic stages of the natural history of this disease: atopic and pulmonary diseases, vasculitis, polyneuritis and intense peripheral eosinophilia. ${ }^{7}$

We prescribed omalizumab to the patient included in this report as he was atopic and presented with IgE-mediated allergic diseases. We recommended this drug because there are reported cases with a favorable response to this drug. ${ }^{7}$ Furthermore, at the time of the decision, we had neither any anti-IL5 drugs, such as mepolizumab, nor enough evidence regarding the effects of mepolizumab on this disease to prescribe it. ${ }^{6,8}$ Therefore, we do not know if the favorable evolution of our patient was due to treatment with omalizumab ${ }^{7}$ or because he only had one of the five EGPA criteria (eosinophilic infiltration in the gastrointestinal tract) that is predictive of a reduced survival rate. ${ }^{5,9}$ However, a recent study ${ }^{10}$ suggests that rituximab may be effective for treating EGPA vasculitis relapses. In addition, mepolizumab is highly effective with a good safety profile in cortico-dependent asthma.

\section{Future directions}

Scientists are encouraged to carry out the future studies on:

- The pathophysiology of the resultant disease states between Th1 and Th2 T-helper lymphocytes thought to respectively govern autoimmune and atopic disease.

- The role of atopy on the pathogenesis of EGPA and its implication in the triggering of symptoms and their severity.

- The application of new approaches to biological-targeted therapies in autoimmunity and the induction of immune tolerance in atopic disease.

\section{Conclusions}

In conclusion, we report an atopic patient who developed a clas- 
sic allergic reaction in childhood and adolescence. In the patient's teens, he began to exhibit multi-system involvement that included severe asthma, gastrointestinal eosinophilic disease, migratory pulmonary infiltrates, vasculitis, and polyneuropathy. By the patient's 31's, the patient met the diagnosis criteria of EGPA. Although it is a rare disease, allergists and other physicians must take EGPA into account to make an early diagnosis.

\section{Acknowledgments}

None.

\section{Ethical statement}

This study was conducted in accordance with the Declaration of Helsinki. The authors obtained the patient's written informed consent for the publication of this case report.

\section{Funding}

None.

\section{Conflict of interest}

None.

\section{Author contributions}

Study design (EGT), analysis and interpretation of data (ARGG), manuscript writing (OMGJ), administration (JBJR), technical or material support (APC), critical revision (RGR). All authors made a significant contribution to this study and have approved the final manuscript.

\section{References}

[1] Pellefigues C. IgE Autoreactivity in Atopic Dermatitis: Paving the Road for Autoimmune Diseases? Antibodies (Basel) 2020;9(3):47. doi:10.3390/antib9030047.

[2] Gomez Torrijos E, García Rodriguez C, Rodriguez J, De la Roca F, Cárdenas R, Alfaya F, et al. Occupational asthma and eosinophilic esophagitis in a patient with egg-bird syndrome. J Investig Allergol Clin Immunol 2015;25(1):61-62.

[3] Gioffredi A, Maritati F, Oliva E, Buzio C. Eosinophilic granulomatosis with polyangiitis: An overview. Front Immunol 2014;5:549. doi:10.3389/fimmu.2014.00549.

[4] Shah A. The pathologic and clinical intersection of atopic and autoimmune disease. Curr Allergy Asthma Rep 2012;12(6):520-529. doi:10.1007/s11882-012-0293-0.

[5] Santos YA, Silva BR, Lira PN, Vaz LC, Mafort TT, Bruno LP, et al. Eosinophilic granulomatosis with polyangiitis (formerly known as Churg-Strauss syndrome) as a differential diagnosis of hypereosinophilic syndromes. Respir Med Case Rep 2017;21:1-6. doi:10.1016/j. rmcr.2017.03.006.

[6] Furuta S, Iwamoto T, Nakajima H. Update on Eosinophilic Granulomatosis with Polyangiitis. Allergol Int 2019;68(4):430-436. doi:10.1016/j.alit.2019.06.004

[7] Cisneros C, Segrelles G, Herráez L, Gonzalez A, Matínez A, Girón R. Churg Strauss syndrome in a patient treated with Omalizumab. J Investig Allergol Clin Immunol 2013;23(7):515-516.

[8] Raffray L, Guillevin I. Treatment of eosinophilic granulomastosis with polyangiitis: a review. Drugs 2018;78(8):809-821. doi:10.1007/ s40265-018-0920-8.

[9] Yılmaz İ, Tutar N, Şimşek ZÖ, Oymak FS, Gülmez İ. Clinical and Serological Features of Eosinophilic and Vasculitic Phases of Eosinophilic Granulomatosis with Poliangiitis: a Case Series of 15 Patients. Turk Thorac J 2017;18(3):72-77. doi:10.5152/TurkThoracJ.2017.16040.

[10] Canzian A, Venhoff N, Urban ML, Sartorelli S, Ruppert AM, Groh M, et al. Use of Biologics to Treat Relapsing and/or Refractory Eosinophilic Granulomatosis with Polyangiitis: data from a European Collaborative Study. Arthritis Rheumatol 2020. doi:10.1002/art.41534. 